\title{
CONTRIBUIÇÕES DA LITERATURA PARA A ENFERMAGEM DE FAMÍLIA NO CONTEXTO RURAL
}

\author{
Gisele Cristina Manfrini Fernandes ${ }^{1}$, Astrid Eggert Boehs ${ }^{2}$
}

\footnotetext{
${ }^{1}$ Doutoranda pelo Programa de Pós-Graduação em Enfermagem (PEN) da Universidade Federal de Santa Catarina (UFSC). Bolsista CNPq. Santa Catarina, Brasil. E-mail: gisamanfrini@terra.com.br

${ }^{2}$ Doutora em Enfermagem. Professora do Departamento de Enfermagem e do PEN/UFSC. Santa Catarina, Brasil. E-mail: astridboehs@hotmail.com
}

RESUMO: Trata-se de um estudo de revisão da literatura de enfermagem que objetivou identificar aspectos do ciclo vital e da saúde da família no contexto rural. Foram analisados 22 artigos, entre 2003 e 2010, obtidos nas bases Medline, Lilacs, BDEnf e Cinahl, selecionados sob critérios de inclusão e de exclusão. Os resultados mostram aspectos da saúde da unidade familiar nas transições típicas das fases do ciclo vital, a relação com os profissionais de enfermagem e implicações da informação/conhecimento de saúde nos comportamentos de cuidado e promoção da saúde familiar. Conclui-se que o cuidado da enfermagem promove a saúde das famílias rurais, ao considerar a cultura, a educação em saúde e o meio ambiente. Reforça a importância da informação e do conhecimento em saúde para a promoção de comportamentos saudáveis nas famílias, aliado ao papel da enfermagem no cuidado centrado na família, com ênfase na educação em saúde.

DESCRITORES: Saúde da família. Enfermagem. Saúde da população rural. Família.

\section{LITERATURE CONTRIBUTIONS TO RURAL FAMILY NURSING}

\begin{abstract}
This is a nursing literature review. It aimed to identify aspects of the life cycle and family health within the rural context. Twenty two articles published between 2003 and 2010 have been analyzed through Medline, Lilacs, BDEnf, and Cinahl databases. They were selected through inclusion and exclusion criteria. Results show health aspects of the family unit during typical transitions of the life cycle, the relationship with nursing professionals, implications about health information/ knowledge related to care behavior, and family health promotion. This study concludes that nursing care promotes rural family health upon considering culture, health education, and the environment. It reinforces the importance of health information and knowledge in order to promote healthy family behaviors, in addition to the nursing role in family-centered care, emphasizing health education
\end{abstract}

DESCRIPTORS: Family health. Nursing. Rural health. Family.

\section{APORTES BIBLIOGRÁFICOS PARA LA ENFERMERÍA FAMILIAR EN EL MEDIO RURAL}

RESUMEN: Se trata de un estudio de revisión bibliográfica de enfermería con el objetivo de identificar los aspectos del ciclo de vida y de la salud familiar en el medio rural. Se analizaron veintidós artículos publicados entre 2003 y 2010, obtenidos en las bases de datos Medline, Lilacs, BDEnf, Cinahl, seleccionados según los criterios de inclusión y exclusión. Los resultados muestran aspectos de la salud familiar en las transiciones, las que son típicas en las fases del ciclo de vida, así como la relación con los profesionales de enfermería, las implicaciones de la información y del conocimiento en salud, relacionados con la atención y la promoción de la salud familiar. Se concluye que la atención de enfermería promueve la salud de las familias rurales al considerar la cultura, la educación para la salud y el medio ambiente, lo que refuerza la importancia de la información y del conocimiento en salud, con el fin de promover comportamientos saludables en las familias, además del papel de la atención de enfermería centrada en la familia y con énfasis en la educación para la salud.

DESCRIPTORES: Salud de la familia. Enfermería. Salud rural. Familia. 


\section{INTRODUÇÃO}

O cuidado de enfermagem está presente em todas as etapas do ciclo vital dos indivíduos e também ao longo da trajetória de vida das famílias. Esta aproximação da enfermagem à família, com a abordagem do desenvolvimento, também denominada de ciclo vital familiar, tem origem com os estudos da sociologia, psicologia e antropologia, sobretudo na segunda metade do século XX. Alguns estudos se tornaram referência teórica sobre família, dentre os quais são apontados os de Paul Glick, Evelyn Duvall, Hill e Rodgers e Aldous. ${ }^{1}$ Recentemente, publicações nas áreas de psicologia ${ }^{2-3}$ e de enfermagem ${ }^{4-7}$ têm contribuído para a socialização desta teoria.

A Estratégia de Saúde da Família (ESF), nas áreas urbana e rural do Brasil, tem reorientado a assistência na atenção básica de saúde, contrária à visão fragmentada do ser humano, e resgatado a prática generalista, para a compreensão do processo saúde-doença, considerando a realidade local, comunitária e familiar. Por sua vez, a compreensão do processo de saúde-doença e o modo como este é representado pelas famílias, especificamente em comunidade rural, ampliam a sua relação com os serviços, com a promoção de cuidados de saúde e a prevenção de doenças. ${ }^{8}$ Tal articulação entre o sistema de cuidado familiar e o sistema de saúde mais amplo, em que se inserem as Unidades de Saúde da Família, instiga a investigação científica, por refletir a lógica de cuidado que repercute deste movimento de reorientação da atenção básica.

Concomitante à expansão da ESF com a ideia da promoção da saúde, a enfermagem à família vem apresentando produção de conhecimento e práticas de cuidado, que, embora ainda uma área em construção, já aponta caminhos de trabalho. A enfermagem tem se aproximado cada vez mais das famílias, tanto na área urbana, quanto na área rural, através da ESF, e tornando-a mais preparada para atuar junto à referida clientela. No entanto, a dificuldade quanto à capacitação de enfermeiros de família se acentua com relação à área rural, uma vez que as escolas de enfermagem têm focado as aulas práticas e estágios na área urbana. É preciso considerar as especificidades de cada contexto e, em essência, a cultura dos sujeitos, ainda que na aparência, os modos de cuidar, nas comunidades rurais, possam ser semelhantes aos desenvolvidos em unidades de saúde urbanas. ${ }^{9}$

Sabe-se que o tema saúde da família, dentro da produção de conhecimento da enfermagem, é bastante amplo e direcionado às dimensões da assistência, do ensino e da pesquisa. Assim, pretendeu-se delimitar a presente revisão a estudos que retratassem as questões da saúde da unidade familiar que vive em contextos rurais e que é atendida pela enfermagem. Este artigo exibe um panorama dos estudos, identifica aspectos da saúde da família no contexto rural relacionados com o ciclo vital familiar e destaca algumas contribuições dos estudos para a prática e pesquisa em enfermagem.

\section{METODOLOGIA}

Foram realizadas revisão e análise da literatura de enfermagem que partiram de um questionamento: o que a enfermagem tem produzido a respeito da saúde da família no contexto rural, relacionada ao ciclo vital familiar? A revisão da literatura foi realizada em algumas etapas, de modo a fornecer uma organização metodológica e rigor ao estudo.

Inicialmente, estabeleceram-se o objetivo do estudo e a sua delimitação, com critérios de inclusão e de exclusão para a seleção dos artigos. Neste sentido, foram utilizadas as bases de dados disponíveis na BIREME (Centro Latino-Americano e do Caribe de Informação em Ciências da Saúde.): o Medical Literature Analysis and Retrieval Sistem on-line (MEDLINE), a Literatura Latino-Americana e do Caribe em Ciências da Saúde (LILACS) e a Base de Dados de Enfermagem (BDEnf) e, além destas, a base Cumulative Index to Nursing and Allied Health Literature (CINAHL). Os critérios de inclusão restringiram a literatura a ser analisada a artigos originais (pesquisa) ou de revisão, somente da enfermagem, com texto completo em três idiomas (português, inglês ou espanhol). As publicações abrangeram o período de 2003 a 2010. Os critérios de exclusão consistiram em outros tipos de publicação, artigos de pesquisa ou revisão sem texto completo e estudos que não contemplassem o objetivo proposto. Utilizaram-se os descritores: saúde da família/family health, família/family, saúde da população rural/rural health, enfermagem familiar/family nursing. Foram selecionados 22 artigos para análise, os quais atenderam aos critérios de escolha estabelecidos.

Para a coleta de dados da literatura, optou-se pela elaboração de um instrumento que contemplava os itens: título, autor(es), ano e periódico de publicação, proposta (objetivo) e população do estudo, método, referencial teórico, principais resultados e contribuições do estudo para a enfermagem. Uma caracterização dos artigos foi apresentada sob frequência absoluta. A análise qualitativa do conteúdo dos resultados e as contribuições dos estudos levaram à descrição de três categorias. 


\section{RESULTADOS}

\section{Panorama dos estudos revisados}

Dentre os autores dos 22 artigos analisados, ao menos um em cada estudo tem formação em enfermagem ou representa alguma instituição de enfermagem. No que concerne ao período de publicação, observa-se, no quadro 1 , que houve um predomínio de artigos sobre o assunto do ciclo vital de família rural, publicados no ano de 2006 $(n=6)$, de $2005(n=3)$ e de $2008(n=3)$. Dentre os periódicos com o maior número de publicações, destacam-se Public Health Nursing $(\mathrm{n}=4)$ e Family $\mathcal{E}$ Community Health $(\mathrm{n}=2)$.

Quadro 1 - Lista de artigos selecionados nas bases de dados Medline, Lilacs, BDEnf e Cinahl, referentes ao período de 2003 a 2010

\begin{tabular}{|c|c|c|c|}
\hline Ano & Título & Autores & Periódico \\
\hline \multirow[t]{2}{*}{2003} & $\begin{array}{l}\text { Describing abuse of pregnant women and their healthcare } \\
\text { workers in rural Appalachia }\end{array}$ & Denham SA & $\begin{array}{l}\text { MCN Am J Matern } \\
\text { Child Nurs }\end{array}$ \\
\hline & Home asthma management for rural families & Horner SD, Fouladi RT & J Spec Pediatr Nurs \\
\hline \multirow{2}{*}{2004} & Providing health education to appalachia populations & $\begin{array}{l}\text { Denham SA, Meyer MG, } \\
\text { Toborg MA, Mande MJ }\end{array}$ & Holist Nurs Pract \\
\hline & $\begin{array}{c}\text { Indicadores socioeconômicos e demográficos de famílias } \\
\text { assentadas no Mato Grosso do Sul }\end{array}$ & $\begin{array}{l}\text { Alvarenga MRM, Rodrigues } \\
\text { FP }\end{array}$ & Rev Enferm UERJ \\
\hline \multirow{3}{*}{2005} & Health-related behavior and adolescent mothers & $\begin{array}{c}\text { Chen MY, James K, Hsu LL, } \\
\text { Chang SW, Huang LH, Wang } \\
\text { EK }\end{array}$ & Public Health Nurs \\
\hline & $\begin{array}{c}\text { Normalization Behaviours of rural fathers living with chronically- } \\
\text { ill children: an Australian experience }\end{array}$ & Peck B, Lillibriddge J & J Child Health Care \\
\hline & $\begin{array}{l}\text { The influence of father absence on the self-esteem and self- } \\
\text { reported sexual activity of rural southern adolescents }\end{array}$ & Hendricks CS, et al. & ABNF J \\
\hline \multirow{6}{*}{2006} & Tobacco prevention in children and cessation in family members & $\begin{array}{l}\text { Tingen MS, Smith TM, Baker } \\
\text { RR, Reyes J, Treiber FA }\end{array}$ & $\begin{array}{l}\text { J Am Acad Nurse } \\
\text { Pract }\end{array}$ \\
\hline & Health risk behavior of rural low-income expectant fathers & $\begin{array}{l}\text { Everett KD, Bullock L, Gage } \\
\text { JD, Longo DR, Geden E, } \\
\text { Madsen R }\end{array}$ & Public Health Nurs \\
\hline & $\begin{array}{c}\text { Rural parents' perceptions of risks associated with their } \\
\text { children's exposure to radon }\end{array}$ & $\begin{array}{l}\text { Hill WG, Butterfield P, } \\
\text { Larsson LS }\end{array}$ & Public Health Nurs \\
\hline & $\begin{array}{c}\text { Does Dixon's integrative environmental health model inform } \\
\text { an understanding of rural parents' perceptions of local } \\
\text { environmental health risks? }\end{array}$ & $\begin{array}{l}\text { Harnish KE, Butterfield P, } \\
\text { Hill WG }\end{array}$ & Public Health Nurs \\
\hline & $\begin{array}{l}\text { Middle-aged appalachians living with diabetes mellitus: a family } \\
\text { affair }\end{array}$ & Lobri-Posey B & $\begin{array}{l}\text { Fam Community } \\
\text { Health }\end{array}$ \\
\hline & $\begin{array}{c}\text { Vulnerabilidade e intoxicação por agrotóxicos em agricultores } \\
\text { familiares do Paraná }\end{array}$ & $\begin{array}{l}\text { Oliveira MLF, Zambrone } \\
\text { FAD. }\end{array}$ & Cienc Cuid Saude \\
\hline \multirow{2}{*}{2007} & $\begin{array}{l}\text { A qualitative study of how families decide to adopt household } \\
\text { smoking restrictions }\end{array}$ & $\begin{array}{l}\text { Kegler MC, Escoffery C, } \\
\text { Groff A, Butler S, Foreman A }\end{array}$ & $\begin{array}{l}\text { Fam Community } \\
\text { Health }\end{array}$ \\
\hline & $\begin{array}{c}\text { Managing family support and dietary routines: type } 2 \text { diabetes in } \\
\text { rural appalachian families }\end{array}$ & $\begin{array}{l}\text { Denham SA, Manoogian } \\
\text { MM, Shuster L }\end{array}$ & Fam Syst Health \\
\hline \multirow{3}{*}{2008} & $\begin{array}{l}\text { Nurse's views of family nursing in community contexts: an } \\
\text { exploratory study }\end{array}$ & Yarwood J & Nurs Prax N Z \\
\hline & $\begin{array}{l}\text { Rural parents' perspectives about information on child } \\
\text { immunization }\end{array}$ & $\begin{array}{l}\text { Miller NK, Verhoef M, } \\
\text { Cardwell K }\end{array}$ & $\begin{array}{l}\text { Rural Remote } \\
\text { Health }\end{array}$ \\
\hline & $\begin{array}{c}\text { Mexican American parent's perceptions of culturally congruent } \\
\text { interpersonal processes of care during childhood immunization } \\
\text { episodes- a pilot study }\end{array}$ & Keller T & $\begin{array}{l}\text { Online J Rural Nurs } \\
\text { Health Care }\end{array}$ \\
\hline \multirow{2}{*}{2009} & $\begin{array}{l}\text { Physical activity within rural families of overweight preschool } \\
\text { children: a pilot }\end{array}$ & Seal N, Yurkovich, E & $\begin{array}{c}\text { Online J Rural Nurs } \\
\text { Health Care }\end{array}$ \\
\hline & $\begin{array}{c}\text { Tradition in transition - intergenerational relations with focus on } \\
\text { the aged and their family members in a South African context }\end{array}$ & $\begin{array}{l}\text { Bohman DM, van Wyk NC, } \\
\text { Ekman SE }\end{array}$ & Scand J Caring Sci \\
\hline \multirow{2}{*}{2010} & $\begin{array}{l}\text { Influential factors of long-term care in a Japanese rural } \\
\text { community examined through interviews of family caregivers }\end{array}$ & Imaiso J, Sasaki U & $\begin{array}{l}\text { J Community Health } \\
\text { Nurs }\end{array}$ \\
\hline & $\begin{array}{l}\text { The transition experience of rural older persons with advanced } \\
\text { cancer and their families: a grounded theory study }\end{array}$ & Duggleby WD, et al. & BMC Palliat Care \\
\hline
\end{tabular}

Texto Contexto Enferm, Florianópolis, 2011 Out-Dez; 20(4): 803-11. 
As populações rurais estudadas representam, na grande maioria, díades familiares ou têm foco em algum integrante: criança, gestante, adolescente, idoso. As famílias que caracterizam a população dos estudos procedem de contextos rurais, em diferentes países (China, Canadá, Austrália, Estados Unidos, Nova Zelândia, Japão, África do Sul e Brasil). Apenas dois dos estudos selecionados retrataram populações rurais brasileiras, do Mato Grosso-MT e do Paraná-PR.

A maior parte dos estudos se valeu de métodos qualitativos $(\mathrm{n}=14)$, um deles o método quali-quantitativo, e os demais, de natureza quantitativa $(n=7)$. Os tipos de estudos qualitativos mais representados foram as pesquisas descritivo-exploratórias $(n=9)$ e as pesquisas fenomenológicas $(\mathrm{n}=4)$. Dos estudos quantitativos, seis eram descritivos (com delimitações variadas, como observacional retrospectivo, correlacional, prevalência de amostra significativa), dois tinham caráter interventivo e o estudo quali/quantitativo também era descritivo.

As técnicas de coleta de dados aplicadas nos desenhos qualitativos foram grupo focal, observação participante, consulta de documentos, entrevista semiestruturada, entrevista em profundidade e uso de instrumento/escala na entrevista. Nos estudos quantitativos, foram utilizados surveys, questionários (com questões abertas e fechadas) e escalas aplicadas em entrevistas pelo telefone, no campo (escola, domicílio) ou por envio de correspondência aos domicílios.

A análise dos dados nos estudos qualitativos seguiu principalmente os métodos de análise temática, análise interpretativa e análise fundamentada nos dados. Nos estudos quantitativos, predominou a análise estatística descritiva. $\mathrm{O}$ amplo uso de programas e de softwares para a organização no registro dos dados e também para a análise evidenciou a utilização de recursos tecnológicos nas pesquisas internacionais.

\section{Aspectos da saúde da família e o ciclo vital familiar}

No que tange aos achados associados a aspectos da saúde da família e do ciclo vital familiar, os estudos exploram comportamentos relacionados à unidade familiar ou a algum membro em especial. Sobressai a relação com cuidado profissional, indicando como a informação/ conhecimento de saúde influencia nas famílias para a prevenção de riscos, a promoção de hábitos saudáveis, a adaptação, o manejo ou a tomada de decisão sobre cuidados específicos.

São descritas as fases do ciclo vital familiar do casal com filhos lactentes e pré-escolares, ${ }^{10-14}$ com filhos na idade escolar, ${ }^{15-17}$ de pais adolescentes, ${ }^{18-19}$ famílias com membros adultos (fase madura) ${ }^{20-22} \mathrm{e}$ famílias com idosos, ${ }^{23-24}$ as quais podem ser identificadas respectivamente, como: fases de aquisição, adolescente, madura e tardia ${ }^{3}$, considerando a etapa de vida dos membros e a idade dos filhos.

Os estudos traduzem transições características do ciclo vital familiar, como a de adolescentes à espera do primeiro filho ${ }^{19}$, de mães adolescentes com filho pequeno dependente do suporte financeiro e presencial das famílias de origem, ${ }^{18}$ da família na situação de doença crônica da criança ${ }^{25}$ ou de doença crônica de adultos, ${ }^{21}$ final de vida ${ }^{22,24}$ e o abuso/violência familiar à mulheres, na infância e durante a gravidez ou o puerpério. ${ }^{26} \mathrm{~A}$ teoria define que transições estão vinculadas à mudança e ao desenvolvimento, acomodando processos vitais contínuos e descontínuos dos seres humanos e podem estar ligadas a determinadas idades dos indivíduos, a eventos inesperados, experiência de doença e uma íntima relação com o ambiente, inclusive o familiar. ${ }^{5}$

Tiveram destaque as transições da fase adolescente pela gravidez e o nascimento do primeiro filho, cujos estudos mostram aspectos da sobreposição da fase de vida individual para a de vida familiar. Tais eventos influenciam nas relações familiares do casal adolescente com a família de origem, nas mudanças dos papéis parentais e nas condições de saúde. ${ }^{18}$ A gravidez na adolescência é um evento que ocasiona estresse na família e também fatores para abuso e violência. ${ }^{26}$

Mudanças nos comportamentos de saúde e a busca de cuidados profissionais podem ser promovidas naturalmente em transições, com a alteração da composição familiar (casamento, divórcio, gravidez e chegada de um bebê), a moradia (compra de uma nova casa, mudar de residência) e a doença ou morte na família. ${ }^{10,22,24}$ De acordo com um estudo, a transição da gravidez na adolescência pode oportunizar a mudança de comportamentos e de estilo de vida de jovens e a promoção da saúde nas futuras famílias. Pais adolescentes comumente adotam poucas práticas saudáveis e, mais frequentemente, optam por comportamentos de risco relacionados ao uso de tabaco, álcool e outras drogas, a má nutrição e pouca atividade física, que são padrões e hábitos precursores de problemas de saúde infantil, como 
a obesidade. ${ }^{21}$ É enfatizada a necessidade de ajudar mães adolescentes a serem saudáveis, atentando aos comportamentos de promoção da saúde. ${ }^{18}$ Por outro lado, conforme revelado em outro estudo, a dificuldade de famílias com crianças escolares obesas em aderir padrões saudáveis de atividade física reflete barreiras ambientais como a falta de opções para o acesso a programas de atividades no meio rural, bem como barreiras pessoais dos pais, como a falta de tempo, pouca energia para atividades além do trabalho e o não reconhecimento da condição de saúde de crianças. ${ }^{17}$ Desta maneira, a atuação da enfermagem familiar na perspectiva do ciclo vital possibilita abordar as crises maturacionais, subsidiando o cuidado no processo de saúde-doença, a prevenção de problemas futuros e a elaboração de estratégias juntamente com a família para a promoção da saúde individual e grupal.

Verifica-se que a presença de crianças motiva as famílias a optarem por comportamentos sinérgicos de prevenção e de proteção da saúde, visto que elas são mais vulneráveis aos riscos do ambiente doméstico e aos efeitos dos comportamentos de risco adotados pelos pais ou adultos. O domicílio é considerado locus na produção tanto de doenças, quanto de bem-estar, e os comportamentos individuais na família causam ambas as condições, refletindo a função da família na saúde individual. ${ }^{20}$ Os resultados de um estudo fornece esta ideia, ao reforçar o papel dos pais em minimizar a exposição passiva ao tabaco. O comportamento de fumar e de ter cigarros disponíveis em casa torna o ambiente doméstico um risco para que crianças iniciem o uso do tabaco e desenvolvam diversas preocupações de saúde. ${ }^{16}$

Em contrapartida, as preocupações com as crianças no ambiente doméstico rural incluem comportamentos preventivos dos pais, como filtragem da água, ventilação da casa, uso de produtos de limpeza não tóxicos, manter materiais perigosos fora do seu alcance e não fumar perto delas. ${ }^{14}$ Neste aspecto, a vulnerabilidade das crianças parece estar ligada também às preocupações dos pais com a pobreza, à falta de suporte social e ao estresse no ajustamento à paternidade. Já no caso de famílias de assentamentos rurais brasileiros, adiciona-se, às precárias condições de vida, o baixo nível de escolaridade. ${ }^{27}$ Ademais, constatou-se que a vulnerabilidade de crianças em famílias de agricultores familiares no Paraná que utilizam agrotóxicos está vinculada à característica do trabalho agrícola compartilhado, ao desempenharem algumas atividades, ficam expostas ao ambiente. ${ }^{28}$
A função de proteger a saúde das crianças ou dos mais jovens influencia na tomada de decisão para alguns comportamentos individuais e familiares, motivada especialmente pelas mulheres e seu papel de promotoras de saúde nas famílias ${ }^{10}$, mas que também pode ser uma reação protetora dos homens. ${ }^{20}$ As famílias estabelecem restrições para proteger as crianças, geralmente por iniciativa das mães. ${ }^{10}$ Todavia, essa noção de territorialidade no domínio doméstico, em torno de interesses familiares, estende-se a muitos assuntos de saúde, em que a figura materna tem função de conservadora da saúde dos membros e de educadora à saúde das crianças..$^{20}$ Contudo, quando a mulher adoece (como o Diabetes Mellitus), pode enfrentar tensões e conflitos para aderir a um plano de alimentação que não seja universalmente dividido entre os membros da família, sobretudo porque elas são as responsáveis primeiras pelas refeições, ou podem conseguir estabelecer rotinas saudáveis e mudar os padrões de alimentação da família em geral. ${ }^{21}$

A descoberta da doença de um integrante pode ser considerada um evento marcante que implica comportamentos para manejo do tratamento e, muitas vezes, a criação, a adaptação e a alteração de rotinas. Um estudo com pais de crianças escolares asmáticas associou a participação da criança no manejo da doença, o engajamento dos pais em comportamentos de prevenção dos sintomas e o seu conhecimento sobre cuidados específicos à severidade da asma. ${ }^{15}$ Outro estudo explora a experiência estressante de pais no processo de aceitação e de adaptação diária à doença crônica das crianças, em que pequenas conquistas relacionadas às habilidades desenvolvimentais dos filhos trazem satisfação como cuidadores. ${ }^{25} \mathrm{O}$ referido estudo exemplifica a dimensão de uma transição familiar estressora nessa etapa de vida familiar e como a manutenção de alguns comportamentos e rotinas parece ajudar na adaptação e na continuidade das tarefas familiares.

Famílias com idosos que necessitam de cuidados paliativos, em contextos rurais, experienciam complexas transições no seu ambiente doméstico, nas funções e nas relações, nas atividades de vida diária e na saúde física e mental dos doentes e dos cuidadores, gerando estresse e incertezas. Conforme o estudo, adaptar-se ao processo de transição requer redefinir o normal da situação, oportunizar a comunicação, buscar informação e suporte na rede. ${ }^{22}$ De outro modo, em famílias rurais japonesas, os membros sentem obrigação em prover o cuidado aos idosos em casa, demonstrando autodependência para essa função 
e uma resistência em aceitar o suporte formal de cuidado. ${ }^{24}$ É importante reconhecer que processos transacionais e culturais não são estáticos e, segundo um estudo realizado com famílias rurais da África do Sul, as gerações mais jovens foram influenciadas pelo impacto da modernização na transição da sociedade tradicional para a industrializada. Isso afetou a tradição do cuidado recíproco entre gerações e nos valores e funções dos idosos. ${ }^{23}$

$\mathrm{Na}$ transição da doença crônica de um adulto, o esforço é direcionado para o alcance do equilíbrio entre as necessidades individuais e as da família ${ }^{29}$ e também para o manejo de múltiplas rotinas, conforme argumentado em uma pesquisa com famílias rurais appalachian. As rotinas de dieta do membro portador de Diabetes Mellitus são confrontadas com as tradições e com os padrões culturais de preparo das refeições e de consumo da comida, resgatando a influência da cultura familiar e de suas implicações ao manejo da doença. ${ }^{21}$ Além dos padrões culturais, os padrões de interação e de comunicação familiar também envolvem a negociação de comportamentos de cuidado à saúde.

O conhecimento das famílias rurais sobre cuidados de saúde específicos, o acesso a serviços de saúde e ao seguro de saúde (no caso de famílias norteamericanas) são fatores determinantes, que influenciam na disposição familiar para cuidar. Enquanto pais de escolares expressam disposição para proteger suas crianças se tiverem informação acerca de riscos e comportamentos preventivos ${ }^{14}$, pais adolescentes à espera do primeiro filho demonstram incongruência de conhecimentos sobre riscos à saúde e atitudes para mudar comportamentos durante a gestação da parceira ${ }^{19}$, sinalizando a relação de tais fatores à etapa do ciclo vital.

A informação também é percebida como um recurso importante para as mães de crianças pequenas, na tomada de decisões a respeito de cuidados de imunização, em que a clareza e a linguagem utilizada pela enfermeira devem facilitar o entendimento sobre as vacinas. ${ }^{11}$ Fazer escolhas informadas para suas crianças e cuidar adequadamente delas após a imunização é primordial ao cuidado feito pelas famílias. ${ }^{12}$ Neste sentido, autores apontam que as mulheres devem se beneficiar de informação sobre saúde e prevenção, de modo a construir habilidades para que a sua função educativa seja mais efetiva nas famílias. ${ }^{20}$

Outros determinantes para a saúde das famílias rurais são citados: o isolamento geográfico de áreas rurais, a acessibilidade limitada aos serviços de saúde, valores de individualidade e ligação comunitária, $^{22}$ falta de consistência na proteção legal e limitadas oportunidades econômicas que incentivam, por exemplo, o problema da violência familiar, ${ }^{26}$ agravando a situação de saúde de indivíduos dentro das famílias.

Com base nos achados descritos, nota-se que a questão rural afeta em parte a saúde da família, considerando os fatores cultural e econômico que aparecem quase como determinantes "universais", ainda que os estudos retratem contextos rurais bastante diversificados. Há um enfoque nos comportamentos individuais e nos da família que influenciam na promoção da saúde de cada membro e da unidade.

\section{A enfermagem e suas habilidades na relação de cuidado às famílias rurais}

A literatura corrobora o papel da enfermagem na interação com a família e indica as habilidades e competências necessárias para o seu cuidado. A prática do cuidado às famílias é um desafio percebido por enfermeiras que atendem em áreas rurais, pelo fato de encararem diversas estruturas, culturas, crenças e valores, exigindo negociação do saber profissional com famílias de complexas dinâmicas e de diferentes gerações. Nos estudos analisados, as enfermeiras assinalam a importância de respeitar a autonomia das famílias e de utilizar uma abordagem de cuidado que considere a unidade familiar e suas partes, entendida também como um "quebra-cabeça". 30:44

Para a conquista de bons resultados da enfermagem familiar, é preciso construir relações efetivas com as famílias, o que requer honestidade, transparência, confiança, integridade, ética, respeito e habilidade..$^{30}$ Algumas habilidades são essenciais para a tomada de decisão acerca dos cuidados com as crianças, ${ }^{11}$ como: o respeito às decisões familiares, a disponibilidade para discutir preocupações com os pais de crianças, a receptividade às dúvidas e questionamentos, o uso de linguagem compreensível e informações claras. Da mesma forma, os profissionais devem estar informados e atualizados.

Segundo um dos estudos, a competência da enfermeira em promover a relação com a família se dá sob duas dimensões: a primeira está em utilizar o tempo para explicar o procedimento; por meio de atitude amigável, minimizar a ansiedade em alguns cuidados; investigar questões ou estar atenta às preocupações das mães. A segunda está no modo com que a enfermeira contribui para que as mães confiem na sua própria habilidade de cuidar dos filhos e de fazer escolhas informadas. ${ }^{12}$ 
É importante que a enfermeira saiba reconhecer as necessidades da família nas diferentes transições, conectando-se ainda às necessidades dos indivíduos, oportunizando que se envolvam no cuidado. ${ }^{22}$ As enfermeiras também reconhecem que o tempo gasto no cuidado das famílias e as estratégias lançadas neste processo dependem do momento de vida que se encontram, por exemplo, se a família está numa fase de transição ou de crise, avalia-se qual a necessidade do suporte profissional..$^{30} \mathrm{O}$ uso da estratégia educativa é um importante recurso de cuidado nas transições pela descoberta de doença crônica na família ${ }^{20-21}$ ou nas situações de imunização das crianças ${ }^{11-12}$ ou durante a gestação de mães adolescentes, ${ }^{18-19}$ ou durante o acompanhamento de pré-natal pelo casal. ${ }^{31}$

Sobre as habilidades das enfermeiras, alguns aspectos profissionais e contextuais são considerados determinantes para o cuidado à família na área rural: a experiência profissional e a maturidade pessoal, os conhecimentos e as habilidades para fazer e articular as interações enfermeira-família, a compreensão do conceito de ruralidade e a implicação desta condição na sensibilidade do cuidado. Também, a relação interdisciplinar para um cuidado integral e não fragmentado às famílias, conhecer a influência da localização geográfica, da condição econômica, do trabalho, da etnicidade e do gênero na saúde das famílias, disposição em se utilizar de estratégias interventivas e de cuidado junto da família e no domicílio. ${ }^{30} \mathrm{O}$ potencial de intervenção pode ser ampliado empregando uma abordagem socioecológica direcionada às necessidades da população rural, o que envolve a participação das famílias e comunidade na alteração de práticas e de políticas de saúde. ${ }^{17}$

Um dos estudos mostrou que, em certas situações, como no caso do abuso e da violência familiar, a educação continuada se faz necessária para o trabalho de enfermeiras nessas famílias. ${ }^{26}$ A enfermagem tem a oportunidade e a responsabilidade de agir preventivamente na violência familiar, informando seus pecientes, sabendo identificar sinais físicos e emocionais na pessoa abusada, desenvolvendo habilidades de comunicação terapêutica pró-ativa na busca de soluções e nas tomadas de decisão para o cuidado. ${ }^{32}$

\section{Contribuições dos resultados para a prática e a pesquisa em enfermagem}

Os autores dos artigos apontam algumas considerações para a prática e a pesquisa de En- fermagem. No tocante à sua prática, o significativo papel das enfermeiras nos contextos histórico, social, cultural e político de cada país torna crucial o desenvolvimento de habilidades para se inteirar com as famílias no cuidado à saúde ${ }^{30}$ Além disso, elas precisam explorar novas habilidades para avaliar dados de saúde ambiental, integrando-as em suas práticas com as famílias rurais. ${ }^{13}$ Tais contribuições nos sinalizam a importância da prática do cuidado de enfermagem em cada contexto, mas também as dimensões cultural, social e ambiental do cuidado e sua relação com a sua saúde e bem-estar. Um dos estudos também enfatiza que, para que as enfermeiras se sintam preparadas e tenham habilidade na avaliação e intervenção de famílias na situação de abuso ou violência, é preciso incluir ou reforçar essa temática na educação de enfermagem e na educação continuada para o trabalho com tal clientela. ${ }^{26}$

A abordagem de enfermagem à família é resgatada como necessidade de os profissionais serem cuidadosos com os pais de crianças cronicamente doentes, respeitando as construções individuais desta experiência e tentando compreender os modos com que buscam normalizar a vida do dia a dia. ${ }^{25}$ Esta ideia de aproximação entre o cuidado de enfermagem e o cuidado das famílias é entendida, teoricamente, como práticas culturalmente congruentes, que ajudam a estabelecer a autoconfiança da família e a confiança nos profissionais, resultando em maior eficácia nas ações preventivas. ${ }^{12}$

Dentre outras práticas de cuidado, os estudos ressaltaram as ações de educação em saúde como instrumento para o cuidado de famílias rurais, ajudando na diminuição das barreiras de conhecimento para a promoção de estilos de vida saudáveis ${ }^{18}$, incentivando e integrando a participação de colaboradores nas comunidades, ${ }^{28}$ ensinando sobre o manejo de cuidados às crianças e nas situações de doença, considerando os recursos disponíveis ${ }^{15}$ e também os valores dentro da cultura..$^{29}$ Os estudos citados contribuem para a importância do papel da enfermagem na educação do cuidado realizado pelas famílias e também à promoção da sua saúde e de ambientes saudáveis.

Quanto às contribuições para pesquisas com famílias rurais, pesquisas instigam elucidar as experiências de pais no cuidado à criança com doenças crônicas específicas, ou em diferentes fases do desenvolvimento. É importante buscar compreender os confrontos das questões da ruralidade à transição da função de cuidador familiar 
e saber se os profissionais promovem o cuidado holístico nesse processo. ${ }^{25}$ Outros estudos apontam a relevância de explorar a presença e a ausência do pai na relação de suporte social e o seu impacto no desenvolvimento dos filhos durante a infância e a adolescência. ${ }^{31}$ Ainda, descobrir como a informação sobre riscos ambientais domésticos pode refletir no conhecimento de saúde nas famílias rurais com crianças e adolescentes. ${ }^{14}$ Trabalhos futuros poderão determinar o papel das enfermeiras nas questões voltadas ao abuso/violência, quando as profissionais são culturalmente semelhantes aos seus pacientes, e aprofundar o conhecimento sobre como as influências culturais afetam o modo com que o abuso é percebido e relatado (linguagem, significados) dentro de grupos culturais. ${ }^{26}$ As sugestões parecem direcionar as lentes dos pesquisadores em enfermagem para os aspectos da cultura das famílias e a relação com a ruralidade, sobre o papel de educador dos profissionais enfermeiros e para a compreensão da influência das famílias no seu desenvolvimento e na promoção da sua saúde.

\section{CONSIDERAÇÕES FINAIS}

Ao concluir esta revisão, reconhece-se que a produção de conhecimento da Enfermagem acerca da saúde da família, no contexto rural, restringiu-se a estudos, em sua maioria, de origem internacional, evidenciando aspectos culturais, relacionados a tais realidades. Em contrapartida, a seleção de poucos estudos brasileiros dentro da referida temática é percebida como um indicativo da escassez de pesquisas na enfermagem que tratem da saúde da família rural, como unidade de cuidado.

Considera-se, portanto, que os artigos selecionados tenham contemplado a expectativa de explorar o tema por oportunizar o conhecimento de aspectos da enfermagem em contextos rurais diferenciados, e até mesmo desconhecidos pela enfermagem brasileira.

Os resultados reforçam a importância da informação e do conhecimento em saúde para a promoção de comportamentos saudáveis nas famílias rurais, aliado ao papel da enfermagem no cuidado centrado na família, com ênfase na educação em saúde. Acredita-se que a presente revisão contribuiu para uma possibilidade de diálogo entre pesquisadores da enfermagem à família e enfermeiros que cuidam de famílias e populações rurais.

Finalmente, com os resultados da presente revisão, pretende-se alertar para a importância de se conhecer mais, em pesquisas futuras, a situação da assistência de enfermagem nas áreas rurais do Brasil e sobre o ensino de enfermagem rural. Os enfermeiros, quando se graduam, muitas vezes se deparam com esta realidade dentro da ESF e enfrentam um cotidiano diferente do vivenciado na área urbana, ao trabalharem com as questões estruturais do sistema de saúde no contexto rural e com as questões culturais que envolvem a saúde das famílias.

\section{REFERÊNCIAS}

1. Hoffmann ACOS, Karkotli APB, Dias SLA, Paes ZP. A teoria do desenvolvimento da família: buscando a convergência entre a teoria e a prática no cotidiano dos profissionais de saúde. Fam Saúde Desenv. 2005 Jan-Abr; 7(1):75-88.

2. Carter B, McGoldrick M. As mudanças no ciclo de vida familiar: uma estrutura para a terapia familiar. Porto Alegre (RS): Artes Médicas; 1995.

3. Cerveny CMO, Berthoud CM, colaboradores. Família e ciclo vital: nossa realidade em pesquisa. São Paulo: Casa do Psicólogo; 1997.

4. Boehs AE. Prática do cuidado ao recém-nascido e sua família, baseado na teoria trans-cultural de Leininger e na teoria do desenvolvimento da família [dissertação]. Florianópolis (SC): Universidade Federal de Santa Catarina. Programa de Pósgraduação em Enfermagem; 1990.

5. Zagonel IPS. O cuidado humano transicional no cuidado de enfermagem. Rev Latino-Am. Enfermagem. 1999 Jul; 7(3):25-32.

6. Manfrini GC. O cuidado às famílias rurais, com base na teoria do desenvolvimento da família [dissertação]. Florianópolis (SC): Universidade Federal de Santa Catarina. Programa de Pósgraduação em Enfermagem; 2005.

7. Hoffmann ACOS. Promovendo a saúde da família na fase da adolescência [monografia]. Florianópolis (SC): Universidade Federal de Santa Catarina; 1999.

8. Gomes LOS, Aguiar MGG. Programa de saúde da família: um espaço para a reconstrução das representações sociais do processo saúde-doença? Rev Bras Enferm. 2006 Set-Out; 59(5):614-9.

9. Budó MLD, Saupe R. Modos de cuidar em comunidades rurais: a cultura permeando o cuidado de enfermagem. Texto Contexto Enferm. 2005 AbrJun; 14(2):177-85.

10. Kegler MC, Escoffery C, Groff A, Butler S, Foreman A. A qualitative study of how families decide to adopt household smoking restrictions. Fam Commununity Health. 2007 Oct-Dec, 30(4):328-41.

11. Miller NK, Verhoef M, Cardwell, K. Rural parents' perspectives about information on child immunization. Rural Remote Health. 2008 AprJun;8(2):863-75. 
12. Keller T. Mexican American parent's perceptions of culturally congruent interpersonal processes of care during childhood immunization episodes- a pilot study. Online J Rural Nurs Health Care. 2008 Sep; 8(2):33-41.

13. Hill WG, Butterfield P, Larsson LS. Rural parents' perceptions of risks associated with their children's exposure to radon. Public Health Nurs. 2006 SepOct; 23(5):392-9.

14. Harnish KE, Butterfield P, Hill WG. Does Dixon's integrative environmental health model inform an understanding of rural parents' perceptions of local environmental health risks? Public Health Nurs. 2006 Sep-Oct; 23(5):465-71.

15. Horner SD, Fouladi RT. Home asthma management for rural families. J Spec Pediatr Nurs. 2003 Abr-Jun; 8(2):52-61.

16. Tingen MS, Waller JL, Smith TM, Baker RR, Reyes J, Treiber FA. Tobacco prevention in children and cessation in family members. J Am Acad Nurse Pract. 2006 Apr; 18(4):169-79.

17. Seal N, Yurkovich E. Physical activity within rural families of overweight preschool children: a pilot. Online J Rural Nurs Health Care. Spring. 2009; 9(1):56-68.

18. Chen MY, James K, Hsu LL, Chang SW, Huang LH, Wang EK. Health-related behavior and adolescent mothers. Public Health Nurs. 2005 Jul-Ago; 22(4):280-8.

19. Everett KD, Bullock L, Gage JD, Longo DR, Geden E, Madsen R. Health risk behavior of rural low-income expectant fathers. Public Health Nurs. 2006 Jul-Ago; 23(4):297-306.

20. Denham SA, Meyer MG, Toborg MA, Mande MJ. Providing health education to appalachia populations. Holist Nurs Pract. 2004 Nov-Dec; 18(6):293-301.

21. Denham SA, Manoogian MM, Shuster L. Managing family support and dietary routines: type 2 diabetes in rural appalachian families. Fam Syst Health. 2007 Mar; 25(1):26-52.

22. Duggleby WD, Penz KL, Goodridge DM, Wilson DM, Leipert BD, Berry PH, et al. The transition experience of rural older persons with advanced cancer and their families: a grounded theory study. BMC Palliat Care. 2010 Apr 26; 9:5.

23. Bohman DM, Van Wyk NC, Ekman SE. Tradition in transition - intergenerational relations with focus on the aged and their family members in a South African context. Scand J Caring Sci. 2009;23:446-455.

24. Imaiso J,Sasaki U. Influential factors of long-term care in a japanese rural community examined through interviews of family caregivers. J Community Health Nurs. 2010; 31:23-31.

25. Peck B, Lillibridge J. Normalization behaviours of rural fathers living with chronically-ill children: an Australian experience. J Child Health Care. 2005 Mar; 9(1):31-45.

26. Denham, SA. Describing abuse of pregnant women and their healthcare workers in rural Appalachia. MCN Am J Matern Child Nurs. 2003 Jul-Ago; 28(4):264-9.

27. Alvarenga MRM, Rodrigues FP. Indicadores socioeconômicos e demográficos de famílias assentadas no Mato Grosso do Sul. Rev Enferm UERJ. 2004 Set-Dez; 12(3):286-91.

28. Oliveira MLF, Zambrone FAD. Vulnerabilidade e intoxicação por agrotóxicos em agricultores familiares do Paraná. Cienc Cuid Saude. 2006; 5(Supl):99-106.

29. Lobri-Posey B. Middle-aged appalachians living with diabetes mellitus: a family affair. Fam Community Health. 2006 Jul-Sep; 29(3):214-20.

30. Yarwood J. Nurses' views of family nursing in community contexts: an exploratory study. Nurs Prax N Z. 2008 Jul; 24(2):41-51.

31. Hendricks CS, Cesario SK, Murdaugh C, Gibbons ME, Servonsky J, Bobadilla RV, Hendricks DL, Spencer-Morgan B, Tavakoli A. The influence of father absence on the self-esteem and self-reported sexual activity of rural southern adolescents. ABNF J 2005 Nov-Dec; 16(6):124-31.

32. Denham SA. Confronting the monster of family violence. Nurs Forum. 1995 Jul-Sep; 30(3):12-9. 